\title{
Chapter 75 \\ Report on the Global Environment \\ Competitiveness of Jordan
}

Jordan is located in the west of Asia, borders on Palestinian, Israel, Iraq, Saudi Arabia and other countries. It's capital Amman and the western mountains are the subtropical Mediterranean climate. The mainly natural resources are phosphate, potassium, copper and so on. It covers 90 thousand square kilometres. It had a population of 6.18 million and domestic production the gross (GDP) of USD 28.8 billion in 2011. Through the evaluation of global environment competitiveness, we can know that the environment competitiveness index of Jordan ranks at 120 among 133 countries.

Fig. 75.1 Contribution of

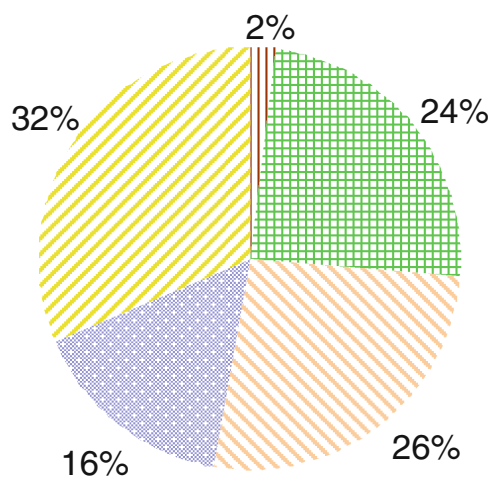

II Resource Environment Competitiveness

\# Ecological Environment Competitiveness Environment Carrying Competitiveness

Environment Management Competitiveness sub-index of GEC

Environment Harmony Competitiveness 


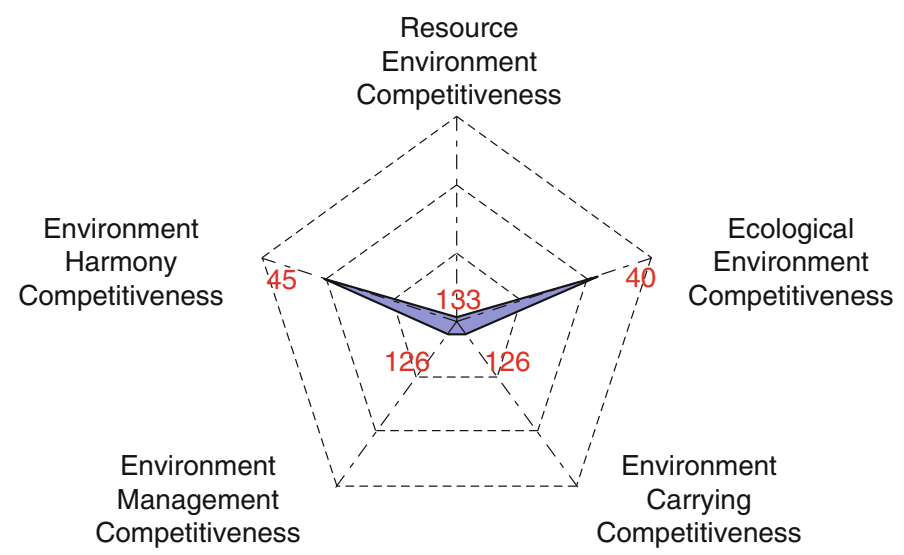

Fig. 75.2 Rank of sub-index of GEC

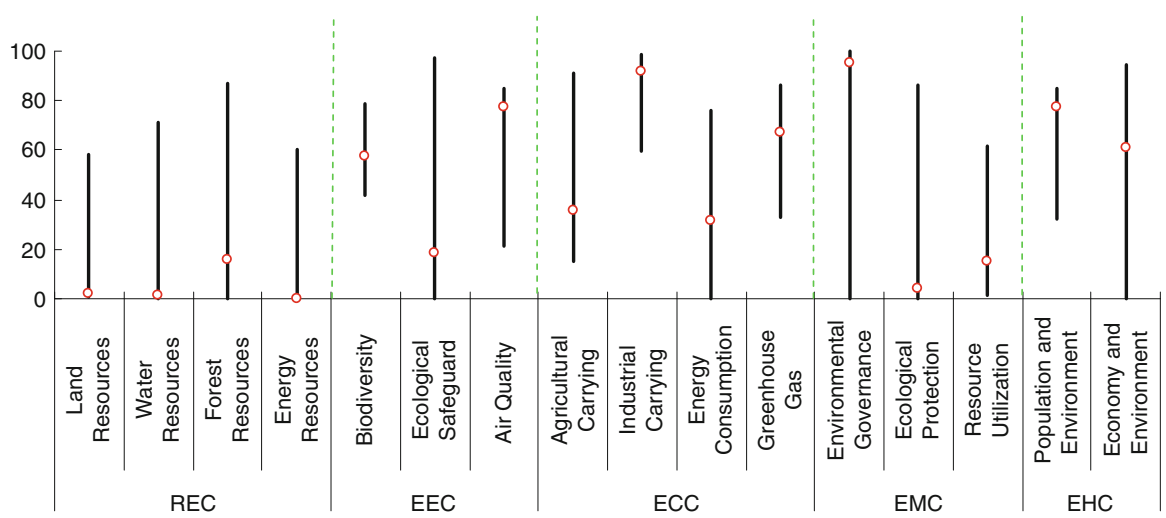

Fig. 75.3 Score and rank of the pillars of GEC

Table 75.1 Score and rank of all indicators of GEC

\begin{tabular}{|c|c|c|c|c|c|}
\hline Indicators & Score & Rank & Indicators & Score & Rank \\
\hline $\begin{array}{l}1 \text { Resource Environment } \\
\text { Competitiveness }\end{array}$ & 4.02 & 133 & $\begin{array}{l}\text { Total internal renewable } \\
\text { water resources }\end{array}$ & 0.39 & 119 \\
\hline 1.1 Land Resources & 2.38 & 129 & 1.3 Forest Resources & 15.55 & 118 \\
\hline Land area per capita & 2.57 & 63 & \multirow{2}{*}{$\begin{array}{l}\text { Growing stock in forest and } \\
\text { other wooded land }\end{array}$} & 50.01 & 118 \\
\hline Percentage of arable land to & 3.19 & 117 & & \multirow{3}{*}{1.29} & \multirow{3}{*}{121} \\
\hline total land area & & & Proportion of land area & & \\
\hline Arable land per capita & 1.32 & 127 & covered by forest & & \\
\hline 1.2 Water Resources & 1.36 & 124 & Forest area per capita & 0.11 & 124 \\
\hline Surface water & 0.14 & 119 & 1.4 Energy Resources & 0.08 & 124 \\
\hline Annual precipitation & 3.79 & 124 & Fossil energy & 0.00 & 64 \\
\hline Groundwater & 1.13 & 107 & Energy production & 0.05 & 124 \\
\hline
\end{tabular}


Table 75.1 (continued)

\begin{tabular}{|c|c|c|c|c|c|}
\hline \multirow{3}{*}{$\begin{array}{l}\text { Indicators } \\
\text { Proportion of combustible } \\
\text { renewables and waste to } \\
\text { total energy consumption }\end{array}$} & Score & Rank & Indicators & Score & Rank \\
\hline & 0.08 & 112 & $\begin{array}{l}\text { Energy consumption per unit } \\
\text { of land area }\end{array}$ & 99.83 & 56 \\
\hline & & & Ratio of clean energy & 2.87 & 83 \\
\hline $\begin{array}{l}\text { Net energy imports of the } \\
\text { energy consumption }\end{array}$ & 0.33 & 122 & consumption & & \\
\hline \multirow{2}{*}{$\begin{array}{l}2 \text { Ecological Environment } \\
\text { Competitiveness }\end{array}$} & 53.76 & 40 & $\begin{array}{l}\text { Elasticity of energy } \\
\text { consumption }\end{array}$ & 14.54 & 28 \\
\hline & 57.51 & 75 & $\begin{array}{l}\text { Elasticity of electric power } \\
\text { consumption }\end{array}$ & 9.92 & 96 \\
\hline Threatened fish species & 93.87 & 36 & 3.4 Greenhouse Gas & 67.43 & 32 \\
\hline $\begin{array}{l}\text { Threatened mammal species } \\
\text { Threatened plant species }\end{array}$ & $\begin{array}{l}92.93 \\
99.94\end{array}$ & $\begin{array}{l}78 \\
22\end{array}$ & $\begin{array}{l}\text { Growth rate of } \mathrm{CO}_{2} \\
\text { emissions }\end{array}$ & 68.84 & 19 \\
\hline \multirow{2}{*}{$\begin{array}{l}\text { GEF benefits index for } \\
\text { biodiversity } \\
2.2 \text { Ecological Safeguard } \\
\text { Terrestrial protected areas }\end{array}$} & 0.40 & 103 & $\begin{array}{l}\text { Growth rate of Methane } \\
\text { emissions }\end{array}$ & 69.72 & 13 \\
\hline & $\begin{array}{r}18.82 \\
4.89\end{array}$ & $\begin{array}{r}74 \\
113\end{array}$ & $\begin{array}{l}\mathrm{CO}_{2} \text { emissions per unit of } \\
\text { land area }\end{array}$ & 99.77 & 71 \\
\hline Marine protected areas & $\begin{array}{l}39.71 \\
77.16\end{array}$ & $\begin{array}{r}7 \\
36\end{array}$ & $\begin{array}{l}\mathrm{CO}_{2} \text { emissions per unit of } \\
\text { energy consumption }\end{array}$ & 30.00 & 101 \\
\hline $\begin{array}{l}\text { Inhalable particles (PM10) } \\
\text { Particulate matter (PM2.5) }\end{array}$ & 78.10 & $\begin{array}{l}69 \\
97\end{array}$ & $\begin{array}{l}4 \text { Environment Management } \\
\text { Competitiveness }\end{array}$ & 34.55 & 126 \\
\hline \multirow{2}{*}{$\begin{array}{l}\text { Index of indoor air pollution } \\
\text { Nitrogen oxides emission } \\
\text { Sulfur dioxide emission }\end{array}$} & 100.00 & 1 & $\begin{array}{l}\text { 4.1 Environmental } \\
\text { Governance }\end{array}$ & 95.10 & 43 \\
\hline & $\begin{array}{l}08.8 / \\
40.71\end{array}$ & $\begin{array}{l}15 \\
62\end{array}$ & $\begin{array}{l}\text { Agricultural chemicals } \\
\text { regulation }\end{array}$ & 95.24 & 20 \\
\hline $\begin{array}{l}3 \text { Environment Carrying } \\
\text { Competitiveness }\end{array}$ & 58.14 & 126 & \multirow{2}{*}{$\begin{array}{l}\text { Percentage of the rural } \\
\text { population with access to } \\
\text { an improved water source }\end{array}$} & 92.00 & 53 \\
\hline 3.1 Agricultural Carrying & 35.61 & 131 & & & \\
\hline $\begin{array}{l}\text { Cereal yield per unit of } \\
\text { arable land }\end{array}$ & 17.95 & 90 & $\begin{array}{l}\text { Percentage of the urban } \\
\text { population with access to }\end{array}$ & 98.00 & 70 \\
\hline $\begin{array}{l}\text { Fertilizer consumption per } \\
\text { unit of arable land }\end{array}$ & 0.08 & 130 & $\begin{array}{l}\text { an improved water source } \\
\text { 4.2 Ecological Protection }\end{array}$ & 3.93 & 130 \\
\hline \multirow{2}{*}{$\begin{array}{l}\text { Annual freshwater } \\
\text { withdrawals for } \\
\text { agriculture per unit of } \\
\text { arable land }\end{array}$} & 94.69 & 96 & $\begin{array}{l}\text { Area of plantation and } \\
\quad \text { afforestation }\end{array}$ & 0.06 & 95 \\
\hline & & & Biome protect & 9.10 & 118 \\
\hline 3.2 Industrial Carrying & 92.03 & 58 & $\begin{array}{l}\text { Overfishing of fishing } \\
\text { resources }\end{array}$ & N/A & N/A \\
\hline $\begin{array}{l}\text { Net exports as a percentage } \\
\text { of GDP }\end{array}$ & 82.54 & 57 & 4.3 Resource Utilization & 14.84 & 131 \\
\hline \multirow{2}{*}{$\begin{array}{l}\text { Electric power consumption } \\
\text { per unit of value added of } \\
\text { industry }\end{array}$} & 86.51 & 98 & $\begin{array}{l}\text { Utilization rate of water } \\
\text { resources }\end{array}$ & 4.03 & 12 \\
\hline & & & Percentage of total internal & 36.37 & 103 \\
\hline $\begin{array}{l}\mathrm{SO}_{2} \text { emissions per unit of } \\
\text { value added of industry }\end{array}$ & 99.63 & 91 & $\begin{array}{l}\text { resources to total water } \\
\text { resources }\end{array}$ & & \\
\hline \multirow{3}{*}{$\begin{array}{l}\text { Annual freshwater } \\
\text { withdrawals for industry } \\
\text { per value added of } \\
\text { industry } \\
\text { 3.3 Energy Consumption }\end{array}$} & 99.44 & 23 & $\begin{array}{l}\text { Percentage of agricultural } \\
\text { land to total land area }\end{array}$ & 13.65 & 122 \\
\hline & & & Percentage of fossil fuel & 5.30 & 108 \\
\hline & 31.79 & 90 & total energy consumption & & \\
\hline
\end{tabular}


Table 75.1 (continued)

\begin{tabular}{|c|c|c|c|c|c|}
\hline Indicators & Score & Rank & Indicators & Score & Rank \\
\hline $\begin{array}{l}5 \text { Environment Harmony } \\
\text { Competitiveness }\end{array}$ & 69.25 & 45 & $\begin{array}{l}\mathrm{CO}_{2} \text { emissions (metric tons } \\
\text { per capita) }\end{array}$ & 91.76 & 67 \\
\hline $\begin{array}{l}\text { 5.1 Population and } \\
\text { Environment }\end{array}$ & 77.37 & 30 & $\begin{array}{l}\text { Energy consumption per } \\
\text { capita }\end{array}$ & 91.71 & 59 \\
\hline $\begin{array}{l}\text { Improved sanitation facilities } \\
\text { (\% of population with }\end{array}$ & 98.00 & 33 & $\begin{array}{l}5.2 \text { Economy and } \\
\text { Environment }\end{array}$ & 61.13 & 86 \\
\hline $\begin{array}{l}\text { access) } \\
\text { Motor vehicles (per 1,000 }\end{array}$ & 81.23 & 71 & $\begin{array}{l}\text { Land resource utilization } \\
\text { efficiency }\end{array}$ & 0.09 & 69 \\
\hline $\begin{array}{l}\text { people) } \\
\text { Renewable internal }\end{array}$ & 0.13 & 125 & $\begin{array}{l}\text { Sulfur dioxide emissions per } \\
\text { unit of GDP }\end{array}$ & 88.21 & 94 \\
\hline $\begin{array}{l}\text { freshwater resources per } \\
\text { capita }\end{array}$ & & & $\begin{array}{l}\text { Carbon dioxide emissions } \\
\text { per unit of GDP }\end{array}$ & 74.29 & 103 \\
\hline $\begin{array}{l}\mathrm{SO}_{2} \text { emissions (metric tons } \\
\text { per capita) }\end{array}$ & 93.20 & 84 & $\begin{array}{l}\text { Energy consumption per unit } \\
\text { of GDP }\end{array}$ & 81.91 & 76 \\
\hline
\end{tabular}

Table 75.2 Rank distribution of the individual indicators of GEC

\begin{tabular}{lllllll}
\hline & $\begin{array}{l}\text { Number } \\
\text { of the } \\
\text { individual } \\
\text { indicators }\end{array}$ & $\begin{array}{l}\text { Rank } \\
1-10\end{array}$ & $\begin{array}{l}\text { Rank } \\
11-30\end{array}$ & $\begin{array}{l}\text { Rank } \\
31-60\end{array}$ & $\begin{array}{l}\text { Rank } \\
61-100\end{array}$ & $\begin{array}{l}\text { Rank } \\
101-133\end{array}$ \\
\hline Sub-index & 14 & 0 & 0 & 0 & 2 & 12 \\
\hline $\begin{array}{l}\text { Resource Environment } \\
\quad \text { Competitiveness } \\
\begin{array}{c}\text { Ecological Environment } \\
\quad \text { Competitiveness }\end{array}\end{array}$ & 11 & 1 & 2 & 2 & 5 & 1 \\
$\begin{array}{c}\text { Environment Carrying } \\
\quad \text { Competitiveness }\end{array}$ & 15 & 0 & 3 & 4 & 6 & 2 \\
$\begin{array}{c}\text { Environment Management } \\
\quad \text { Competitiveness }\end{array}$ & 10 & 0 & 2 & 2 & 1 & 5 \\
$\begin{array}{c}\text { Environment Harmony } \\
\text { Competitiveness }\end{array}$ & 10 & 0 & 1 & 1 & 6 & 2 \\
Total & 60 & 1 & 8 & 9 & 20 & 22 \\
\hline
\end{tabular}

Open Access This chapter is distributed under the terms of the Creative Commons Attribution Noncommercial License, which permits any noncommercial use, distribution, and reproduction in any medium, provided the original author(s) and source are credited. 